This item was submitted to Loughborough's Research Repository by the author.

Items in Figshare are protected by copyright, with all rights reserved, unless otherwise indicated.

\title{
Wet forming of concentrated nano-BaTiO3 suspensions
}

PLEASE CITE THE PUBLISHED VERSION

PUBLISHER

(C) Elsevier

VERSION

AM (Accepted Manuscript)

LICENCE

CC BY-NC-ND 4.0

\section{REPOSITORY RECORD}

Santacruz, Isabel, M. Isabel Nieto, J.G.P. Binner, and Rodrigo Moreno. 2009. "Wet Forming of Concentrated Nano-batio3 Suspensions". figshare. https://hdl.handle.net/2134/4681. 
This item was submitted to Loughborough's Institutional Repository (https://dspace.lboro.ac.uk/) by the author and is made available under the following Creative Commons Licence conditions.

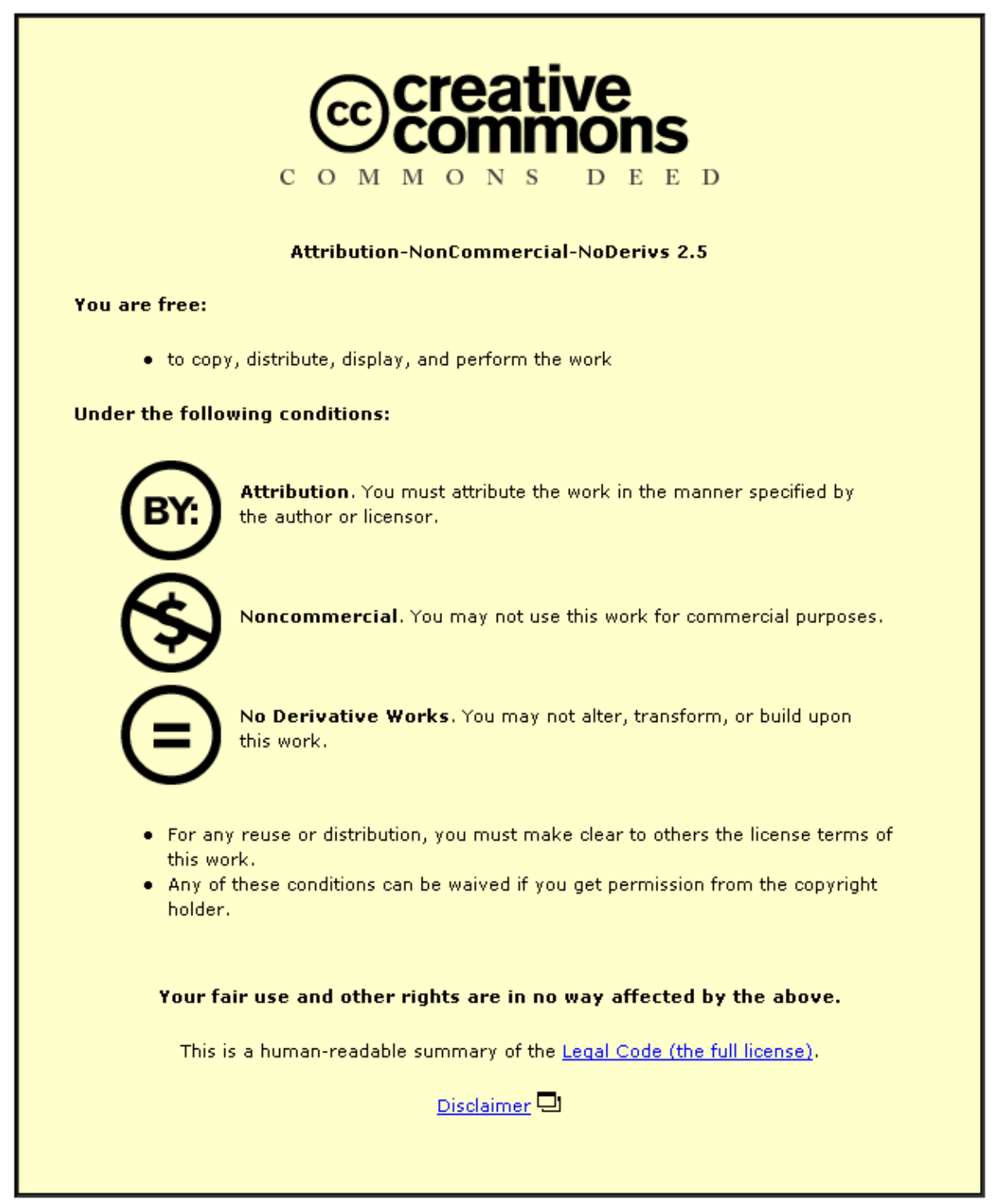

For the full text of this licence, please go to: http://creativecommons.org/licenses/by-nc-nd/2.5/ 


\title{
Wet forming of concentrated nano $\mathrm{BaTiO}_{3}$ suspensions
}

\author{
Isabel Santacruz ${ }^{1,2, *}$, M. Isabel Nieto ${ }^{2}$, Jon Binner ${ }^{1}$, Rodrigo Moreno ${ }^{2}$ \\ ${ }^{1}$ IPTME, Loughborough University, Loughborough, LE11 3TU Leicestershire, UK \\ ${ }^{2}$ Instituto de Cerámica y Vidrio, CSIC, C/ Kelsen nº 5, Campus de Cantoblanco, 28049 Madrid, \\ Spain.
}

\begin{abstract}
Nanostructured materials are currently being studied extensively due to their unusual physical and mechanical properties. One of the most important dielectric materials is barium titanate $\left(\mathrm{BaTiO}_{3}\right)$. Capacitors made of this material offer excellent frequency characteristics, high reliability, high breakdown voltage and excellent volumetric efficiency. It is believed that these properties may be significantly improved by achieving a structure at the nano scale.

In this work, nano $\mathrm{BaTiO}_{3}$ powders have been formed into complex-shaped green bodies using gelcasting as colloidal forming route. This procedure has demonstrated to be very effective for shaping submicron-sized powders and in this manuscript the achievement of this technique for the shaping of nano-sized powders is evaluated. The first step was the preparation of stable concentrated suspensions of the nanopowders through the optimization of the quantity of dispersant and time of homogenization. This was found to depend on the method of manufacture of the different commercial nanopowders investigated. Bulk components were then manufactured using aqueous gelcasting involving polysaccharides that gel on cooling. The performance of this consolidation technique for obtaining dense green bodies from the $\mathrm{BaTiO}_{3}$ nanopowders will be discussed.
\end{abstract}

Keywords: suspensions, shaping, $\mathrm{BaTiO}_{3}$

\section{Introduction}

The recent interest in nanostructured materials is due to their unusual properties. Colloidal processing allows the production of complex-shaped parts with decreased number and size of pores and higher reliability. Gelcasting is a bulk fabrication process for manufacturing near-net shaped components.

Thermogelling polysaccharides like agar, agarose and carrageenan have demonstrated a high efficiency

\footnotetext{
*Corresponding author:

Tel: +34 917355840, Fax: +34 917355843

E-mail: cruz@icv.csic.es,m.i.santacruz@lboro.ac.uk
}

at low concentration ${ }^{1}$ when they are dissolved into a warm slurry, which is then cast or injected in a mould cavity cooled below the glass transition temperature, $\mathrm{T}_{\mathrm{g}}^{2}$.

Barium titanate $\left(\mathrm{BaTiO}_{3}\right.$ or $\left.\mathrm{BT}\right)$ is used extensively in the production of multilayer ceramic capacitors. It has been reported that barium titanate is not thermodynamically stable in acidic aqueous solutions ${ }^{3,4}$ because $\mathrm{Ba}^{2+}$ ions are leached out of the powder, which results in a titanium enriched surface layer. The $\mathrm{Ba}^{2+}$ present in the solution also arises from impurities of $\mathrm{BaCO}_{3}$ present in the $\mathrm{BaTiO}_{3}$ powder. The concentration of dissolved $\mathrm{Ba}^{2+}$ ions increases as the $\mathrm{pH}$ decreases below $7^{5}$. Due to the 
complexity of the system, some authors prefer to use organic $\mathrm{BaTiO}_{3}$ suspensions ${ }^{6}$.

The current interest in using different types of ultrafine, nanosized powders puts a high demand on the suspension processing and control of the forming process. Ammonium polyacrylates have been found to be very useful in the stabilization of micron, submicron ${ }^{7-9}$ and nanosized $\mathrm{BaTiO}_{3}$ suspensions ${ }^{10,11}$ at basic values of $\mathrm{pH}$. Recently, Boschini et al. ${ }^{12}$ found that the combination of both an acrylic polyelectrolyte and a strong quaternary base, viz. tetramethylammonium hydroxide, TMAH, yielded greater stability to concentrated $\mathrm{BaZrO}_{3}$ suspensions than the polyelectrolyte or base on their own.

This work aims to study the dispersion of commercial nanosized powders of $\mathrm{BaTiO}_{3}$ and the preparation and characterization of concentrated suspensions for shape forming pieces by gelcasting with thermogelling polysaccharides.

\section{Experimental procedure}

\subsection{Powder characterization}

Two commercial nanosized $\mathrm{BaTiO}_{3}$ powders (HPB-1000, TPL Inc., USA, and Nanostructured \& Amorphous Materials, Inc., USA) were used as starting materials. They are referred to as P1 and P2 respectively. The characteristics of the powders are shown in Table I.

Table I. Properties of the powders.

\begin{tabular}{|c|c|c|c|c|c|}
\hline Powder & $\begin{array}{c}\mathrm{S}_{\mathrm{s}} \\
\mathrm{m}^{2} / \mathrm{g}\end{array}$ & $\begin{array}{c}\rho \\
\mathrm{g} / \mathrm{cm}^{3}\end{array}$ & $\begin{array}{c}\mathrm{d}_{\mathrm{BET}} \\
\mathrm{nm}\end{array}$ & $\begin{array}{c}\text { Wt loss } \\
(\mathrm{TG}) \\
600^{\circ} \mathrm{C}\end{array}$ & $\mathrm{Ba} / \mathrm{Ti}^{*}$ \\
\hline $\mathrm{P} 1$ & 17.0 & 5.5 & 64 & $\sim 0.7 \%$ & $0.996-1.004$ \\
\hline $\mathrm{P} 2$ & 31.2 & 4.3 & 45 & $\sim 5 \%$ & $0.995-1.010$ \\
\hline
\end{tabular}

* Data provided by the manufacturer

The surface area was measured by one point $\mathrm{N}_{2}$ adsorption (Monosorb, Quantachrome, USA), and the density with a He picnometer (Quantachrome, USA). The particle size values shown in the table were calculated using the values obtained by BET and were confirmed using a field emission gun scanning electron microscope (Leo 1530 VP, Elektronenmikroskopie GmbH, Germany). The zeta potential of BT in aqueous suspensions without any dispersant was measured as a function of $\mathrm{pH}$ with an AcoustoSizer II (Colloidal Dynamics, Australia). Thermogravimetric, TGA, and X-Ray diffraction analysis were performed on both powders.

\subsection{Suspensions preparation}

Aqueous suspensions were prepared by adding tetramethylammonium hydroxide $^{12}$, TMAH, supplied by Aldrich-Chemie (Germany) as a $25 \mathrm{wt} \%$ aqueous solution to distilled water generating a $\mathrm{pH}$ up to 11.0. Subsequently 2.0 and $3.0 \mathrm{wt} \%$ of an ammonium salt of polyacrylic acid (Duramax D3005, Rohm \& Haas, USA) was added as deflocculant prior to the addition of the powder in concentrations of 70 and $80 \mathrm{wt} \%$ (28 and $40 \mathrm{vol} \%$ ). Suspensions were prepared by mechanical agitation with helices and then ultrasonically homogenized using a $400 \mathrm{~W}$ sonication probe, UP400S (Hielscher Ultrasonics GmbH, Germany), for up to $10 \mathrm{~min}$.

A commercially available agar (Grand Agar, Hispanagar S.A., Burgos, Spain) was used as the gelling agent. Solutions of agar were prepared to concentrations of $6 \mathrm{wt} \%$ by heating in a pressure vessel in order to achieve the high temperatures, $110^{\circ} \mathrm{C}$, required for its full dissolution ${ }^{2}$. The rheological behavior of the suspensions was studied through control rate measurements using a rheometer (Haake RS50, Germany) with a double-cone and plate system.

The influence of the volume fraction of solids, the dispersant concentration and the time of ultrasonic mixing on the flow behavior were studied.

\subsection{Green samples}

Barium titanate green bodies were obtained by gelcasting. For preparing the gelcasting suspensions, the well-dispersed BT suspensions (80 wt\%, 40 vol\%) were heated to $50^{\circ} \mathrm{C}$ where the rheological properties were not affected by significant evaporation. The agar solution was then added at this temperature in a concentration of $0.5 \mathrm{wt} \%$ with regard to dry solids. The green densities were measured by the Archimedes' method using mercury. The green fracture surfaces were observed by scanning electron microscopy, SEM (Zeiss DSM40, Germany).

\section{Results and discussion}

\subsection{Powder characterization}

The properties of the precursor powders such as agglomeration, binder content and $\mathrm{Ba} / \mathrm{Ti}$ ratio, which are key aspects in the preparation of the aqueous BT suspensions and determine the quality 
of the final samples, result from the powder synthesis method. Fig. 1 shows the micrographs of the two as-received powders. P1 consists of hard spherical granules with diameters ranging from 5 to $40 \mu \mathrm{m}$, typical of a spray dried powder. The primary particle size is about 50-60 nm. P2 has similarly sized primary particles but consists of smaller and more irregularly shaped agglomerates that are more loosely formed.

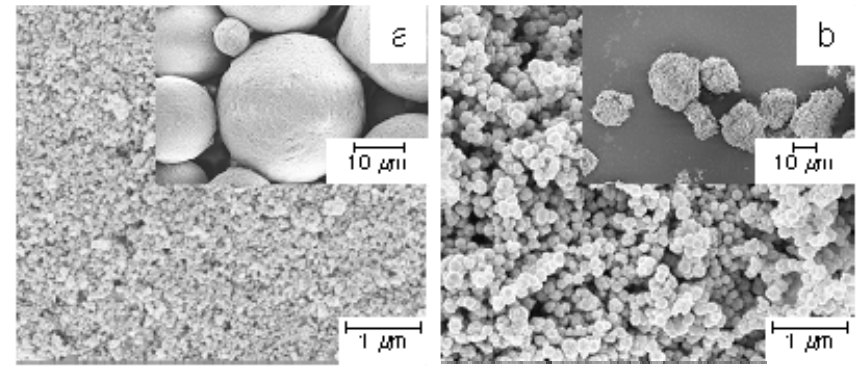

Fig. 1 Micrographs of the as-received P1 (a) and P2 (b) powders.

The TG analysis of P2, fig. 2, shows a larger binder content than for P1, $\sim 5$ and $\sim 0.5 \mathrm{wt} \%$, respectively, Table I. The same table shows an unusually low density value for powder P2. Since the same value was obtained for the powder as-received and after calcination at $350^{\circ} \mathrm{C}$ for $0.5 \mathrm{~h}$, the value was clearly independent of the binder content. X-ray diffraction experiments were performed on the calcined P2, fig. 3, in order to understand why the density is so low. This revealed a cubic $\mathrm{BaTiO}_{3}$ structure with displaced peaks ${ }^{13}$ that indicate larger lattice parameters and hence confirm the open structure, which explains the lower density.

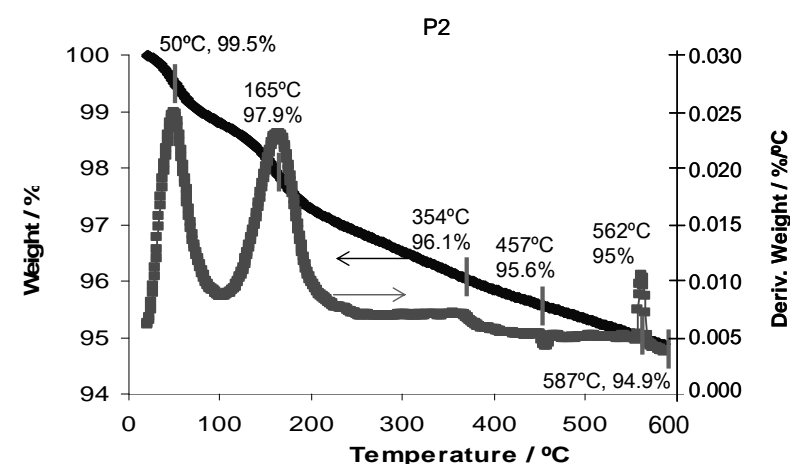

Fig. 2. TGA curves from P2 powder.

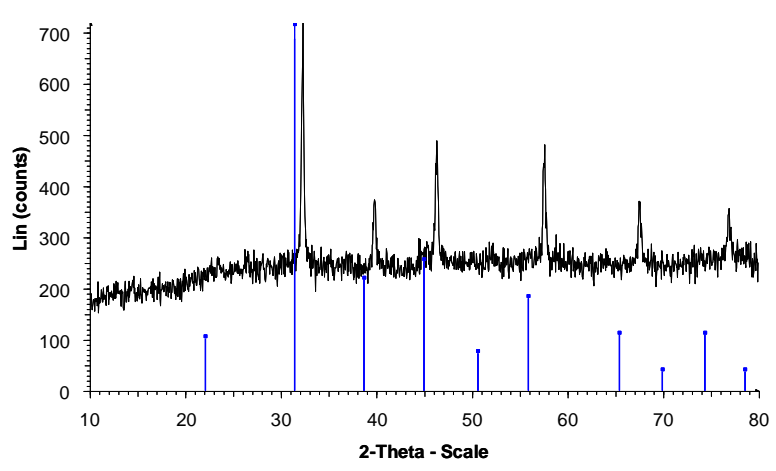

Fig. 3. X-Ray Diffraction of calcined P2 powder.

\subsection{Suspensions preparation}

The variation of zeta potential with $\mathrm{pH}$ was measured via continuous titration from their initial $\mathrm{pH}$ values, 9 and 8.5 for $\mathrm{P} 1$ and $\mathrm{P} 2$ respectively, to $\mathrm{pH} 12$, and from there on to $\mathrm{pH} 2$ using dilute $\mathrm{P} 1$ and P2 suspensions prepared in $0.01 \mathrm{M} \mathrm{KCl}$ solutions without any dispersant. Isoelectric points of the powders were found to occur at $\mathrm{pH} 9.5$ and 8.3 for P1 and P2 respectively. This difference is probably due to the slight differences in the $\mathrm{Ba} / \mathrm{Ti}$ ratio ${ }^{14}$. The IEP values for $\mathrm{BaTiO}_{3}$ reported in the literature differ widely, from $\sim 4$ to $\sim 10$, although a "true" isoelectric point was found at 6.5 by extrapolation to zero $\mathrm{BaCO}_{3}$ in $\mathrm{BaTiO}_{3}{ }^{14}$. The results for $\mathrm{P} 2$ powder are plotted in fig. 4. At basic $\mathrm{pH}$ values the absolute value of zeta potential is not large enough to stabilize the suspension, hence the addition of some deflocculant is required.

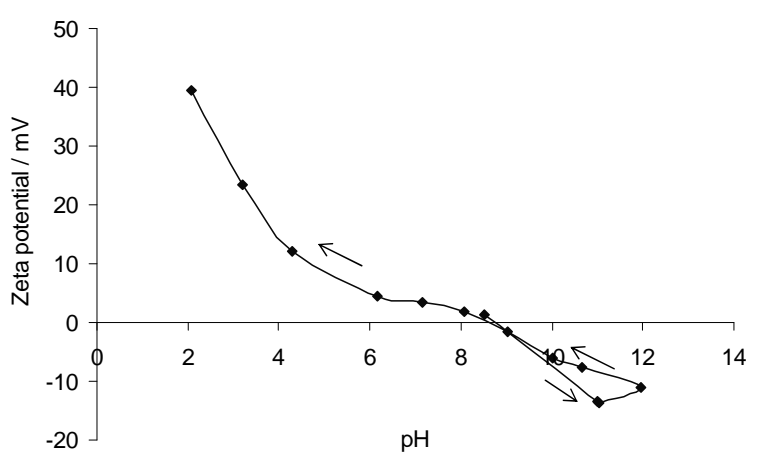

Fig. 4. Evolution of the zeta potential of $\mathrm{P} 2$ with $\mathrm{pH}$.

In the case of acidic $\mathrm{pH}$ values, higher zeta potential values were observed as a result of the electrostatic repulsion force created by the large positive zeta potential developed at the 
particle-solution interface. Since $\mathrm{BaTiO}_{3}$ powder behaves like both a simple insoluble oxide and partially soluble inorganic salt, the zeta potential was determined both by the $\mathrm{H}+$ and $\mathrm{Ba}^{2+}$, where the contribution made by $\mathrm{Ba}^{2+}$ to the surface charge was considerable and was most marked at lower $\mathrm{pH}$ values ${ }^{14}$. Since the concentration of dissolved $\mathrm{Ba}^{2+}$ ions increases as $\mathrm{pH}$ decreases at $<7$, changing the surface chemistry of $\mathrm{BaTiO}_{3}$ and increasing the $\mathrm{pH}$ of resulting suspension, it is not recommended to work in the acidic $\mathrm{pH}$ range.

Concentrated suspensions, 70 wt\%, were prepared from both powders using $\mathrm{TMAH}^{12}$ and ammonium polyacrylate ( $2.0 \mathrm{wt} \%$ active matter). The rheological behavior of both suspensions without ultrasound treatment was quite similar, being slightly thixotropic for P1 suspension, as shown in fig. 5 . When these suspensions were homogenized by using an ultrasound probe for a few minutes, their rheological behavior became very different; in the case of the P1 suspension, the viscosity decreased as expected, due to the better stabilization achieved when the agglomerates were broken down. However, in the case of P2, the viscosity and the thixotropy of the suspension increased and the suspension became very viscous.

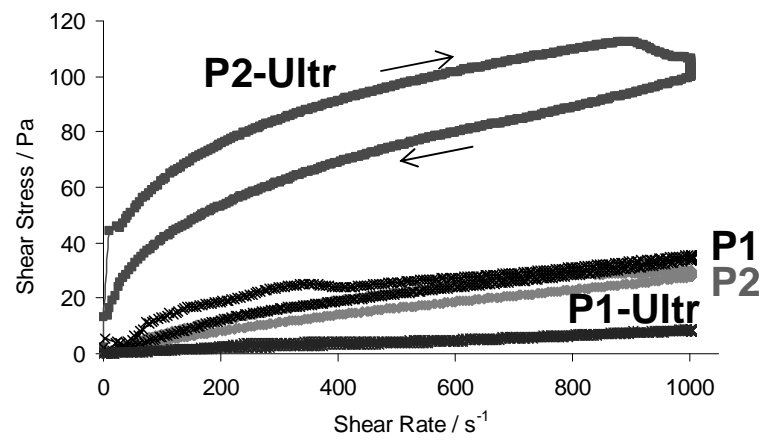

Fig. 5. Flow curves of $70 \mathrm{wt} \% \mathrm{P} 1$ and $\mathrm{P} 2$ suspensions with and without ultrasound.

It is believed that this behavior is related to the presence of a significant amount of organics, which makes it difficult to achieve stabilization as a result of poor dispersant adsorption. Hence the preparation of more concentrated P2 suspensions was limited. These organics are very sensitive to the strong heating and diffusion effects of the ultrasound probe. For this reason and according to the TG curve, figure 2, P2 was calcined at $350^{\circ} \mathrm{C}$ for $0.5 \mathrm{~h}$ before the preparation of further suspensions. To be sure that no large agglomerates were formed after the heat treatment, the calcined $\mathrm{P} 2$ powder was observed by
FEG-SEM, fig. 6. Although a similar image to the as-received powder was observed, the calcined P2 powder allowed the preparation of more concentrated suspensions, $80 \mathrm{wt} \%$, which were impossible to obtain without calcination.

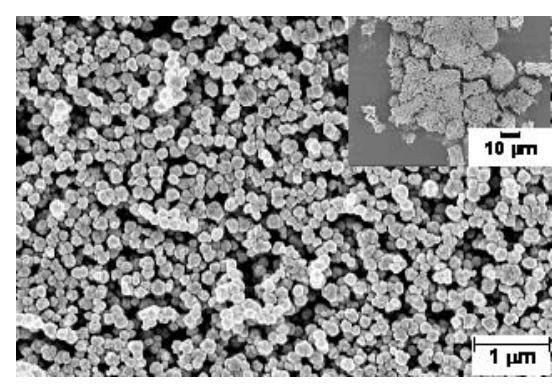

Fig. 6. Micrographs of the calcined P2 powders.

The dispersant content was optimized for both powders. For P1 and P2, maximum values of zeta potential were found when 2.0 and $3.0 \mathrm{wt} \%$ of polyacrylate, respectively, were added to the suspensions. The flow curves of the $80 \mathrm{wt} \% \mathrm{P} 1$ suspensions with different dispersant contents prepared with TMAH and without ultrasound are plotted in fig. 7. The lowest shear stress values were found at any shear rate when 2.0 and $3.0 \mathrm{wt} \%$ deflocculant, respectively, was added.

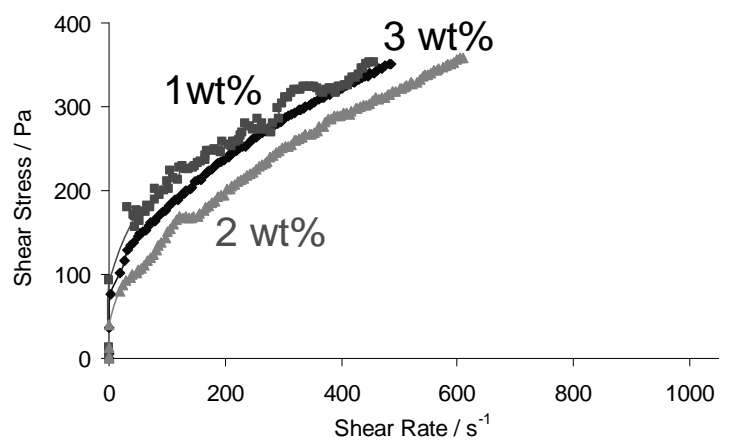

Fig. 7. Flow curves of $80 \mathrm{wt} \% \mathrm{P} 1$ suspensions with different polyacrylate contents.

A decrease in $\mathrm{pH}$ was also found for $\mathrm{P} 2$ suspensions with TMAH when increasing the dispersant content in the suspension, viz. $\mathrm{pH}$ values of 10.1, 9.8 and $9.6 \pm 0.1$ were measured for 1.0, 2.0 and $3.0 \mathrm{wt} \%$ polyacrylate additions respectively. Similar values were found for P1.

Since the ultrasound probe was demonstrated to be very effective in the stabilization of suspensions, 
different ultrasound times were applied to the same volume of $80 \mathrm{wt} \%$ suspensions of either $\mathrm{P} 1$ or P2 dispersed with 2.0 and 3.0 wt\% dispersant, respectively. Flow curves were measured for both P1 and calcined P2 suspensions. The viscosity values of the up-curves taken at a shear rate of $100 \mathrm{~s}^{-1}$ are plotted in fig. 8. It can be seen that longer times of ultrasonic mixing produce lower viscosity suspensions and hence increased stability, with a lowest value after $10 \mathrm{~min}$. For calcined P2 suspensions, the viscosity was reduced by more than a factor of 3 after 10 minutes of ultrasound meanwhile for P1 suspensions the viscosity was reduced by more than a factor of 7 after 8 minutes of ultrasound. Also, it can be observed that the suspension prepared from P1 displays a lower viscosity than the calcined P2 suspension at any ultrasound time. The presence of the remaining agglomerates of P1 suggests that they are hard enough to remain unbroken after ultrasonication, indicating that they may be retained in the green samples.

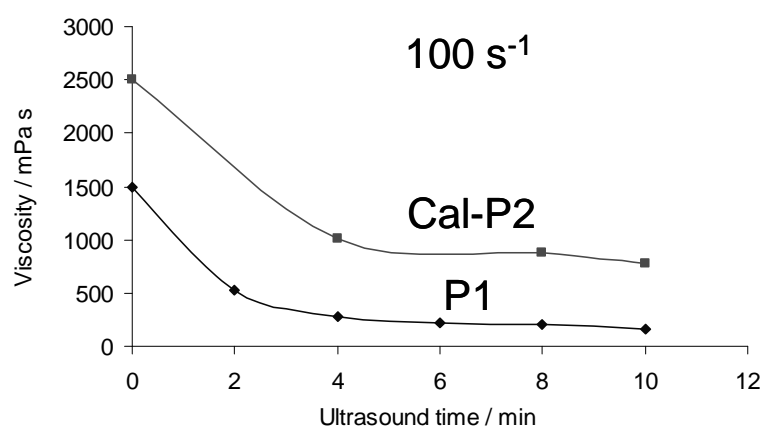

Fig. 8. Viscosity of $80 \mathrm{wt} \% \mathrm{P} 1$ and $\mathrm{P} 2$ suspensions as a function of ultrasound time.

\subsection{Green samples}

Green samples were obtained by gel casting from stable suspensions of the $\mathrm{P} 1$ and calcined $\mathrm{P} 2$ powders prepared at $80 \mathrm{wt} \%$ solids loading after 10 minutes of ultrasonication. Green fracture surfaces of the samples at different magnifications are shown in fig. 9. Spherical agglomerates similar to those observed in the as-received powder can be seen in figs. 9 a and b, confirming that they could not be broken after 10 minutes of ultrasonication. The green density was $56 \%$ of the measured powder density. A previous study demonstrated that these P1 agglomerates also persist in die pressed samples, even after micronising the powder for several minutes and applying 500 MPa of pressure ${ }^{15}$. On the other hand, the gelcast samples prepared from the calcined P2 powder had a higher green density, $60 \%$ of measured density of powder, and a homogeneous microstructure without large agglomerates, figs. 9 c and d, as observed previously for the gelcasting of other ceramic powders ${ }^{16}$.

\section{Conclusion}

Stable nano- $\mathrm{BaTiO}_{3}$ suspensions have been prepared with solids loadings as high as $80 \mathrm{wt} \%$ by dispersing nanopowders with 2.0 and $3.0 \mathrm{wt} \%$ of ammonium polyelectrolyte (active content), for P1 and P2 respectively, in combination with TMAH and the use of an ultrasound probe for mixing times of up to $10 \mathrm{~min}$. In the case of powder P2, the use of ultrasound leads to a higher viscosity as a result of a high binder content, so the powder needed to be calcined if concentrated suspensions were to be prepared successfully.

Bulk components were manufactured by aqueous gelcasting using concentrated agar solutions (6 wt\%) up to a final content of agar of 0.5 $\mathrm{wt} \%$ on a dry solids basis. Unbroken agglomerates, similar to those observed in the as received powder, were present in the green samples manufactured with powder P1, whereas homogeneous green bodies without large agglomerates were obtained for the calcined P2 powder, according to the green density results.

Gelcasting of polysaccharides resulted an excellent alternative to avoid the extremely slow rates and size limitations which appear in the slip casting of nanoceramics.

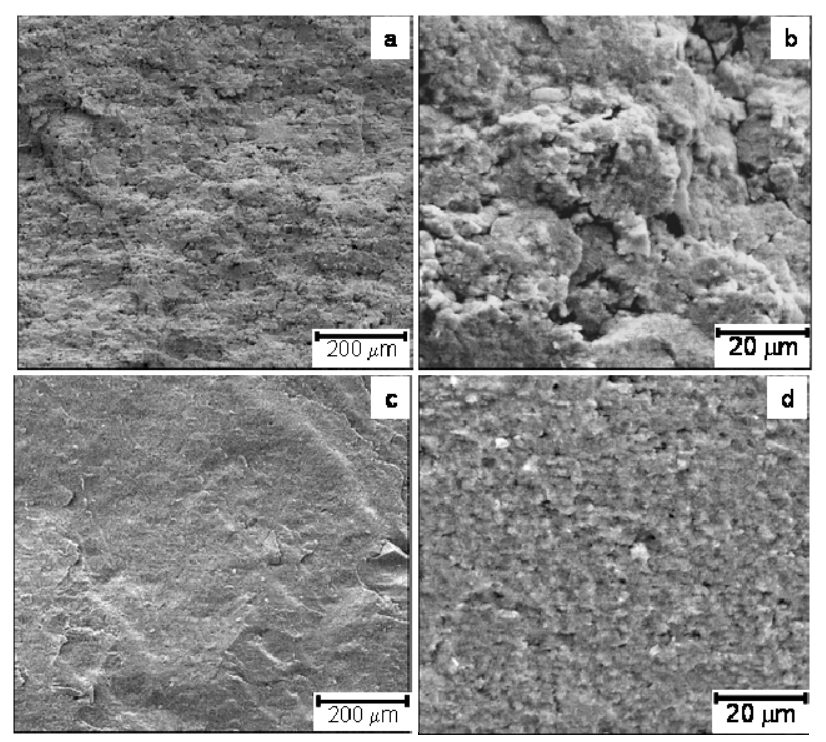

Fig. 9. Microstructures of the green samples prepared by gel casting from 80 wt\% P1 (a and b) and P2 (c and d) suspensions. 


\section{Acknowledgements}

Dr. Santacruz thanks the Spanish Education and Science Ministry for the granting of a postdoctoral fellowship (EX2004-1012). This work has been partially supported by Spanish MEC (CICYT MAT2003-00836).

\section{References}

1. Santacruz I., Nieto M.I. and Moreno R., Alumina bodies with near-to-theoretical density by aqueous gelcasting using concentrated agarose solutions, Ceram. Int., 2005, 31(3) 439-45.

2. Santacruz, I., Nieto, M.I., Moreno, R., Ferrandino, P., Salomoni, A. and Stamenkovic, I., Aqueous injection moulding of porcelains, J. Eur. Ceram. Soc., 2003, 23, 2053-60.

3. Hean, J.H. and Wang, H.R., Effects of solids loading, $\mathrm{pH}$, and polyelectrolyte addition on the stabilization of concentrated aqueous $\mathrm{BaTiO}_{3}$ suspensions, J. Am. Ceram. Soc., 2000, 83(2), 77-80.

4. Chiang, C.W. and Jean, J.H., Effects of barium dissolution on dispersing aqueous barium titanate suspensions, Mater. Chem. Physics, 2003, 80, 647-55.

5. Blanco-Lopez, M.C, Rand, B., and Riley, F.L., The properties of aqueous phase suspensions of barium titanate, J. Eur. Ceram. Soc., 1997, 17, 81-87.

6. Bergström, L., Shinozaki, K., Tomiyama, H., and Mizutani, N., Colloidal processing of a very fine $\mathrm{BaTiO}_{3}$ powder - Effect of particle interactions on the suspensions properties, consolidation, and sintering behaviour, J. Am. Ceram. Soc. 1997, 80, 291-300.

7. Chen, Z.C., Ring, T.A., and Lemaitre, J., Stabilization and processing of aqueous $\mathrm{BaTiO}_{3}$ suspension with polyacrylic acid, J. Am. Ceram. Soc., 1992, 75, 3201-208.
8. Gomez-Yañez, C., Balmori-Ramirez, H., and Martinez, F., Colloidal processing of $\mathrm{BaTiO}_{3}$ using ammonium polyacrylate as dispersant, Ceram. Int., 2000, 26, 609-16.

9. Tseng, W.J. and Li, S.Y., Rheology of colloidal $\mathrm{BaTiO}_{3}$ suspension with ammonium polyacrylate as dispersant, Mater. Sci. Eng. A, 2002, 333, 14-19.

10. Shen, Z.G., Chen, J.F., Zou, H.K,. and Yun, J., Dispersion of nanosized aqueous suspension of barium titanate with ammonium polyacrylate, $J$. Colloid Interface Sci., 2004, 275, 158-64.

11. Shen, Z.G., Chen, J.F., Zou, H.K. and Yun, J., Rheology of colloidal nanosized $\mathrm{BaTiO}_{3}$ suspension with ammonium polyacrylate as dispersant, Colloids and Surfaces A: Physicochem. Eng. Aspects, 2004, 244, 61-66.

12. Boschini, F., Rulmont, A., Cloots, R., and Moreno, R., Colloidal stability of aqueous suspensions of barium zirconate, J. Eur. Ceram. Soc., 2005, 25, 3195-201.

13. Kim, Y., Jung, J.K., and Ryu, K.S., Structural study of nano $\mathrm{BaTiO}_{3}$ powder by Rietveld refinement, Mater. Res. Bull., 2004, 39, 1045-53.

14. Blanco-Lopez, M.C., Rand, B., and Riley, F.L., The isoelectric point of $\mathrm{BaTiO}_{3}$, J. Eur. Ceram. Soc., 2000, 20, 107-18.

15. Santacruz, I., Wells, C. and Binner, J., Microwave two stage sintering of nanostructured barium titanate, Proc. 10th Int. Conf. on Microwave and High Frequency Heating, 2005. Bologna, Italy.

16. Santacruz, I., Baudín, C., Nieto, M.I. and Moreno, R., Improved green strength of gelcast alumina bodies through addition of concentrated polysaccharide, Adv. Eng. Mater., 2004, 6(8), 672-76. 
